\title{
Efficacy and Tolerability of a Shampoo Containing Broad-Spectrum Cannabidiol in the Treatment of Scalp Inflammation in Patients with Mild to Moderate Scalp Psoriasis or Seborrheic Dermatitis
}

\author{
Colombina Vincenzi ${ }^{a}$ Antonella Tosti ${ }^{b}$ \\ aNigrisoli Hospital Bologna, Bologna, Italy; ${ }^{b}$ Dr. Phillip Frost Department of Dermatology and Cutaneous Surgery, \\ University of Miami Miller School of Medicine, Miami, FL, USA
}

\author{
Keywords \\ Scalp psoriasis - Seborrheic dermatitis - Topical treatment . \\ Broad-spectrum cannabidiol shampoo - Dermoscopy . \\ Trichoscopy · Androgenetic alopecia
}

\begin{abstract}
Introduction: Scalp inflammation is commonly associated with scalp psoriasis or seborrheic dermatitis. It can aggravate the progression of androgenetic alopecia and cause troublesome itching and burning. Here, we evaluate the efficacy of a shampoo containing $0.075 \%$ broad-spectrum cannabidiol in 50 subjects with mild to moderate scalp psoriasis or seborrheic dermatitis. Methods: Inflammation was assessed by evaluating the density of twisted/glomerular or arborizing vessels by trichoscopy using a 6-point scale at baseline and day 14 . Symptoms of itching/burning sensation, clinical evaluation of erythema/scaling, overall tolerability, and subject satisfaction were evaluated using 10-point scales. $\boldsymbol{R e}$ sults: There were significant reductions in arborizing vessel/ twisted capillary inflammation and scaling by day 14 . Severity scores reduced from $2.3 \pm 0.1,2.6 \pm 0.1$, and $3.6 \pm 0.1$, respectively, to $0.5 \pm 0.05,0.8 \pm 0.05$, and $0.6 \pm 0.05$ (all $p<$ 0.0001 ). Symptom scores of itching and burning also re-
\end{abstract}

duced significantly from $6.9 \pm 0.1$ to $4.5 \pm 0.1$ to $1.5 \pm 0.05$ and $1.0 \pm 0.05$ (both $p<0.0001$ ). Severity of erythema and scaling was also significantly reduced from $5.5 \pm 0.1$ and 7.0 \pm 0.1 , respectively, to $1.3 \pm 0.05$ and $1.6 \pm 0.05$ (both $p<$ $0.0001)$. Tolerability and subject satisfaction were both excellent. There were no significant differences in treatment effect, tolerability, or subject satisfaction between men and women. Discussion/Conclusion: Replacing current shampooing practices with a broad-spectrum cannabidiol-containing shampoo significantly reduces both severity and symptoms of scalp inflammation within 2 weeks, with excellent tolerability and treatment satisfaction in subjects with mild to moderate scalp psoriasis or seborrheic dermatitis.

(c) 2020 S. Karger AG, Basel

\section{Introduction}

Oxidative stress and scalp inflammation cause telogen effluvium, which affects the health of the follicle and could trigger and aggravate progression of androgenetic alopecia [1-4].

Scalp inflammation is commonly associated with scalp psoriasis or seborrheic dermatitis (SD). Scalp psoriasis

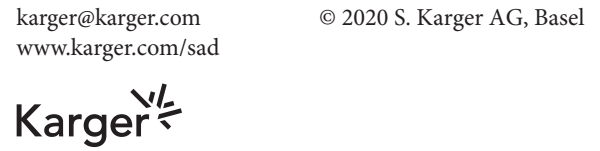

Colombina Vincenzi

Nigrisoli Hospital

Viale Ercolani 9

IT-40138 Bologna (Italy)

colombina.vincenzi@unibo.it 
can be a cause of significant physical and social distress, with $97 \%$ of affected individuals reporting interference with their daily lives [5]. Patients are often symptomatic and complain of itching and burning [6]. Itching was identified as the most bothersome symptom in $43 \%$ of respondents to a telephone survey [7]. Hair loss is also very common in the author's experience.

Psoriasis is also a very common skin disease. Although women generally have less severe psoriasis than men, specifically in scalp psoriasis severity is similar, perhaps due to gender-specific differences in hair growth care and styling [8]. SD is one of the most common inflammatory dermatoses in middle-aged people and is particularly prevalent in men and people with light and dry skin [9].

Trichoscopy is proven to be a noninvasive, cost-effective, and useful diagnostic method to assess the severity of inflammatory changes in these conditions. In scalp psoriasis, trichoscopy shows red dots at low magnification and twisted/glomerular capillary loops at higher magnifications [10]. In SD, it shows an increased number of arborizing vessels [11]. Here, we use trichoscopic evaluation of the scalp to assess the efficacy of an anti-inflammatory shampoo (Revita;CBD, DS Laboratories) in treating scalp inflammation due to mild/moderate scalp psoriasis or SD. In addition to a number of ingredients known to have beneficial effects in scalp conditions (ketoconazole, rooibos tea, taurine, caffeine, apple polyphenol, cysteine, spin traps, carnitine tartrate, biotin, ornithine, emu oil, methylsulfonylmethane, and ornithine), the shampoo contains as its main ingredient the phytocannabinoid, (-)-cannabidiol (CBD), which exerts sebostatic and anti-inflammatory effects on the pilosebaceous unit [12].

\section{Methods}

Before initiating the study, subjects were required to give written consent. Subjects who met the inclusion criteria were eligible for the study. The inclusion criteria were those of either gender, aged 18-65 years, with mild to moderate scalp psoriasis or SD. Severity of disease was established through clinical evaluation of scaling and erythema and subjective assessment of symptoms of itching and burning. The exclusion criteria were scarring alopecia, alopecia areata, pregnancy/breast feeding, history of hair transplant, and a known allergy or sensitivity to the study medication or any of its components.

Trichoscopic evaluation of the scalp to assess inflammation was undertaken with a video dermatoscope (FotoFinder Systems $\mathrm{GmbH}$, Bad Birnbach, Germany), with $\times 20$ and $\times 50$ magnification photographs, using a 6 -point scale where $0=\leq 10,1=10-25,2=$ $25-50,3=50-75,4=75-100$, and $5=100 \%$ of the involved field of dermoscopy patient images for arborizing vessels, twisted capil- laries, and scales as described previously [13]. Clinical evaluation of scaling and erythema utilized a 10-point scale (where $1=$ none).

Subjects provided an insight into symptoms of itching and burning by answering a number of questions. Their overall severity of itching and burning was assessed using a 10-point scale (where $1=$ none). Subsequent reduction of itching and burning was also assessed using a 7 -point scale (where $-3=$ great decrease and $+3=$ great increase).

Subjects were instructed to use the study shampoo over a 14day period without changing their shampooing frequency. Changes in disease and symptom severity were again assessed at the end of the study.

In addition, the subjects were assessed for tolerability and treatment satisfaction using a 10-point subjective scale. The subjects were asked how satisfied they were with therapy, and their views on treatment attributes such as smell, consistency, and ease of application using a similar scale. Gender data were also analyzed.

\section{Statistical Analysis}

Data are presented as mean \pm SEM. Comparisons between groups were made using Student's $t$ test. GraphPad Prism 6 software for OSX was used to run the analysis. Differences were considered significant at $p \leq 0.05$.

\section{Results}

Participants in the study comprised 24 males and 26 females, aged 18-61 years (mean age 38 and 46 years, respectively). Seven subjects were categorized as mild and 43 subjects as moderate disease. Twenty-two subjects had scalp psoriasis and 28 subjects SD.

At baseline, the mean severity scores for arborizing vessels, twisted capillaries, and scales were $2.3 \pm 0.1,2.6 \pm$ 0.1 , and $3.6 \pm 0.1$, respectively. The mean severity scores for erythema and scaling were $5.5 \pm 0.1$ and $7.0 \pm 0.1$, respectively. Subjective assessment scores for itching and burning at baseline were $6.9 \pm 0.1$ and $4.5 \pm 0.1$, respectively.

Following 2 weeks' treatment with the study shampoo, severity scores for arborizing vessels, twisted capillaries, and scales reduced to $0.5 \pm 0.05,0.8 \pm 0.05$, and $0.6 \pm 0.05$, respectively (Fig. 1: all $p<0.0001$ ). Severity scores for erythema and scaling reduced to $1.3 \pm 0.05$ and $1.6 \pm 0.05$, respectively (Fig. 2: both $p<0.0001$ ).

Scores for itching and burning at week 2 were $1.5 \pm$ 0.05 and $1.0 \pm 0.05$, respectively (both $p<0.0001$ ). The mean scores for the reduction in itching and burning were $2.7 \pm 0.05$ and $2.7 \pm 0.05$, respectively, where $2=$ moderate reduction and $3=$ great reduction (Fig. 3 ). Subject assessment mean scores for tolerability and overall satisfaction at the end of the study were both 9.0 (Fig. 4: range $7-10)$. There were no side effects reported. The 


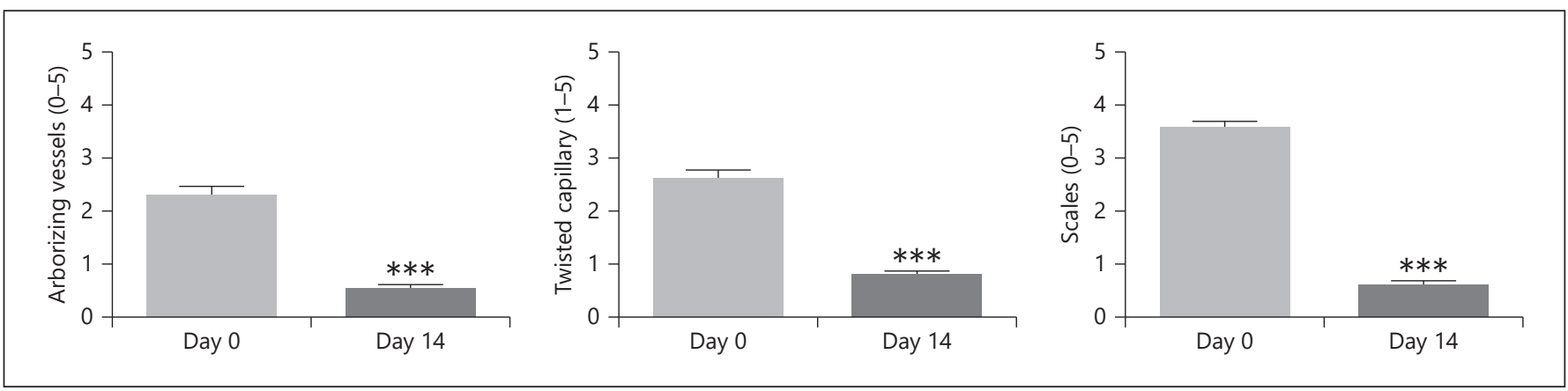

Fig. 1. Effect on mean scores (SEM) following 2 weeks' treatment. Scores for arborizing vessels, twisted capillary, and scales assessed by videodermoscopy. ${ }^{* * *} p<0.0001$.

Fig. 2. Clinical evaluation. Effect on mean scores (SEM) of erythema and scaling following 2 weeks' treatment. Scores using a 10 -point scale. ${ }^{* * *} p<0.0001$.

Fig. 3. Subject self-assessment. Effect on mean scores (SEM) for itching and burning following 2 weeks' treatment and reduction in itching/burning. Scores using the 10 -point scale and reduction assessed using the 7 -point scale. ${ }^{* * *} p<0.0001$.
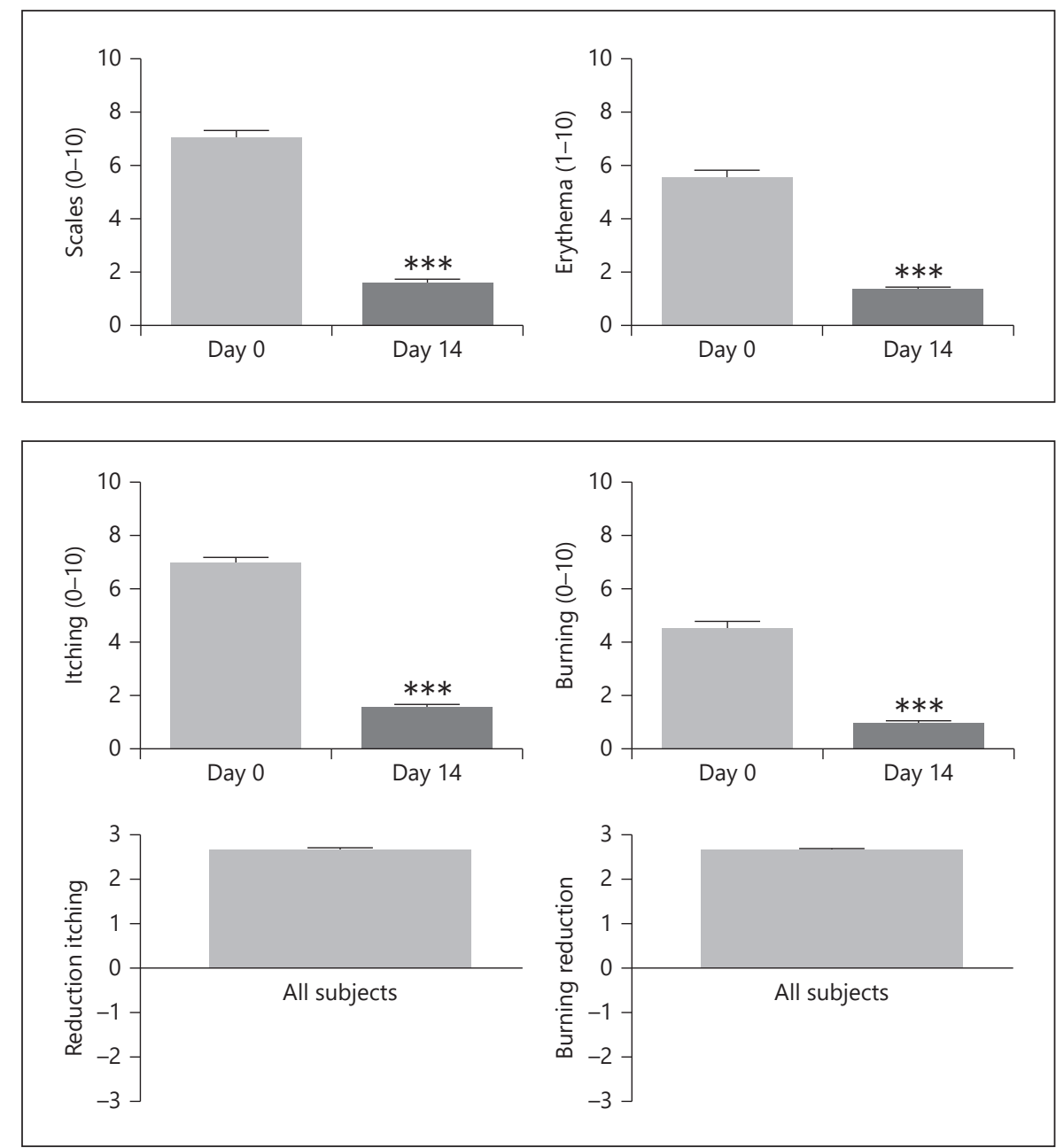

mean scores for smell, consistency, and ease of application were 8.7, 8.1, and 9.2, respectively (out of a maximum score of 10). Representative examples of the efficacy of the study shampoo are shown in Figure 5.

Broad-Spectrum Cannabidiol Shampoo for Inflammatory Scalp Disorders

\section{Gender Assessment}

At baseline, disease severity as assessed by dermoscopy was similar in males (mean scores for arborizing vessels, twisted capillaries, and scales of 1.2, 1.2, and 1.8, respectively) compared with $1.0,1.4$, and 1.8 , respectively, in 
Fig. 4. Tolerability and subject satisfaction at week 2 . Scores using the 10 -point scale.
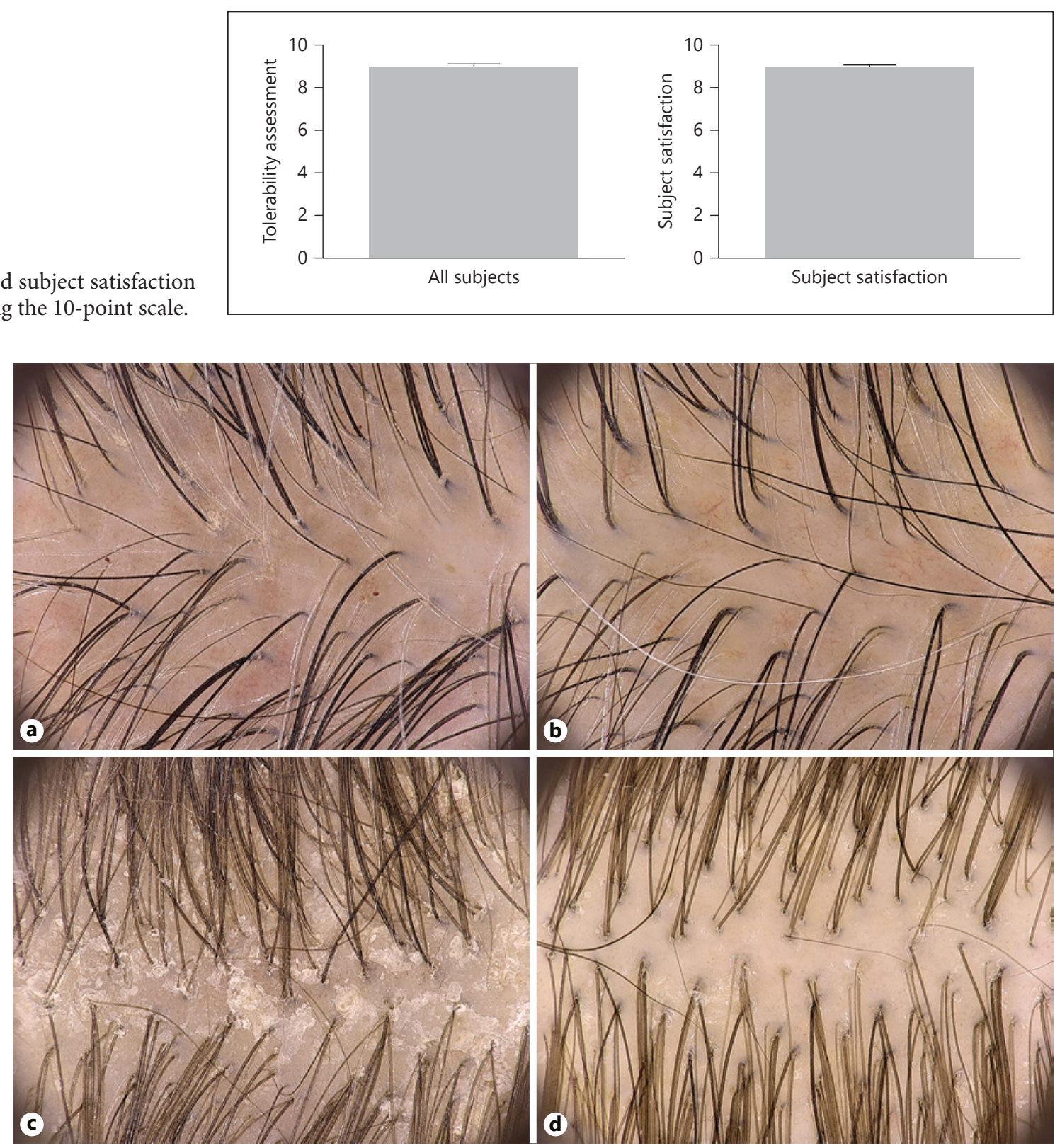

Fig. 5. Representative examples of efficacy of study shampoo using videodermoscopy in subjects with SD (a, b) and scalp psoriasis $(\mathbf{c}, \mathbf{d})(\times 20$ magnification). $\mathrm{SD}$, seborrheic dermatitis.

females. Clinical evaluation of erythema and scaling was also similar (mean scores 2.7 and 3.4 vs. 2.8 and 3.6, respectively). Subject assessment scores for itching and burning were slightly higher in females (mean scores 3.3 and 2.1 vs. 3.6 and 2.4 , respectively).

At week 2, the benefits of treatment were similar across gender (Fig. 6). There were no significant gender differences in terms of reduction in itching or burning, tolerability, or subject satisfaction.

\section{Discussion}

Scalp psoriasis and SD are both very common dermatoses, with significant distress and impaired quality of life. Psoriasis of the scalp presents earlier and more frequently than that of other sites, with itching being the most bothersome symptom affecting every patient to some degree. There is a positive correlation between the severity of the lesions and itch [14]. SD, which affects approxi- 


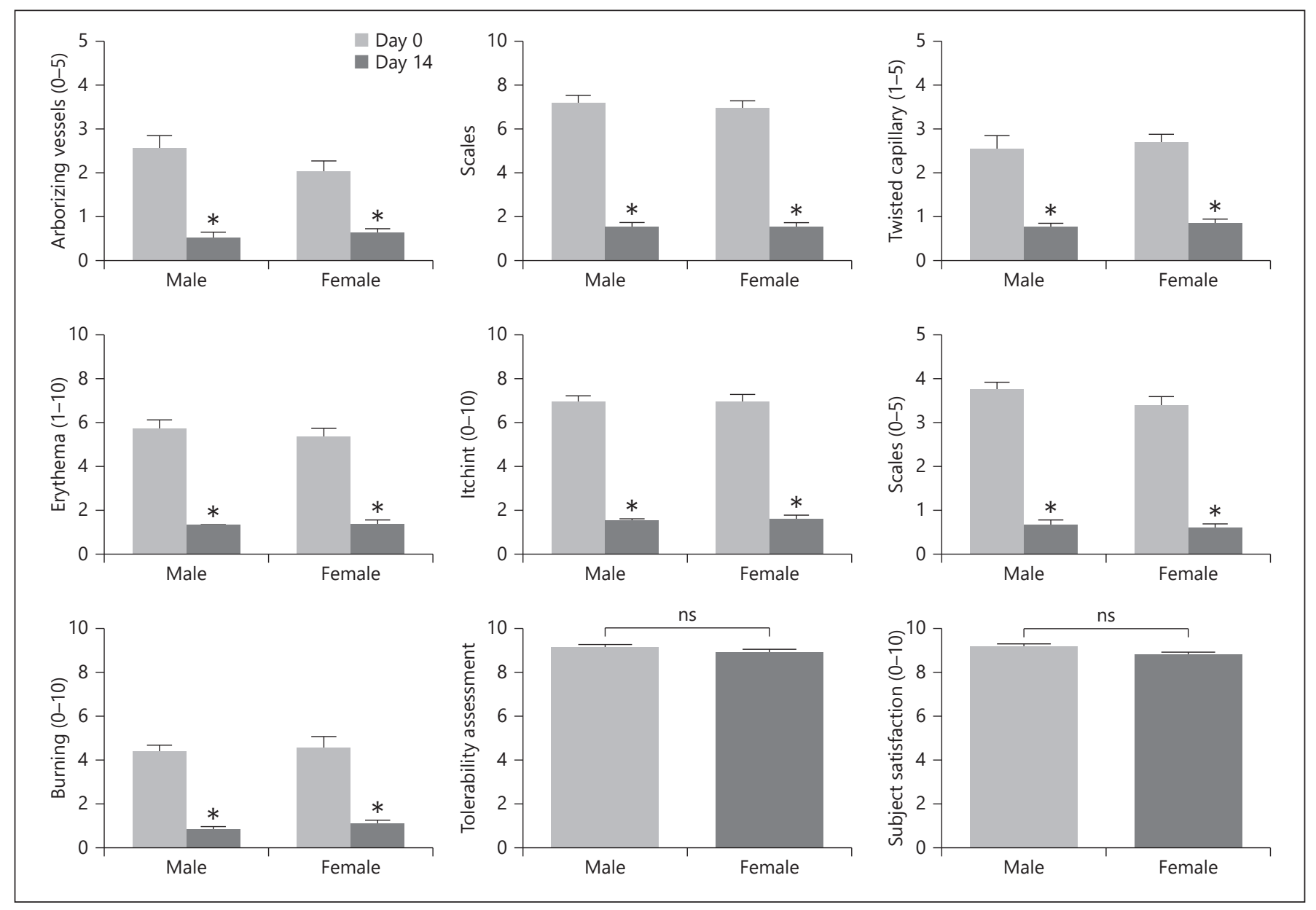

Fig. 6. Gender differences in efficacy, tolerability, and subject satisfaction as assessed by videodermoscopy, clinical evaluation, and subject evaluation. ${ }^{*} p<0.01,{ }^{* * *} p<0.0001$.

mately $16 \%$ of the adult population, is the most common cause of scalp itching. Both conditions can cause telogen effluvium and accelerate progression of androgenetic alopecia.

The diseases can be distinguished using trichoscopy [15]. Patients with scalp psoriasis present with red dots at low magnification and twisted/glomerular vessels at high magnification. Additional features include red loops, hairpin vessels, white scales, and punctate hemorrhages. In patients with $\mathrm{SD}$, trichoscopy shows an increased number of arborizing vessels. They both represent pathologic conditions in which oxidative stress and inflammation are commonly detected [1]. The inflammatory process in SD is mediated by fungal metabolites from Malassezia spp. [16], specifically free fatty acids released from triglycerides. There are clear signs of oxidative stress as indicated by perturbed surface and systemic antioxidant enzyme levels [17] and observations from surface samples of elevated lipid peroxidation [18]. Overgrowth of the scalp with Malassezia spp. has also been described in scalp psoriasis [19]. Indicators of oxidative stress in psoriasis include antioxidant enzymes [20], oxidized proteins [21, 22], and oxidized lipids [23].

Shampoo treatments are the most commonly used means of managing hair and scalp conditions and have proven to be effective in the treatment of psoriasis [2] and SD [24-26]. They exert their benefits through controlling scalp Malassezia levels, and the resultant oxidative stress and inflammation [27].

Here, we studied the efficacy and tolerability of a shampoo uniquely formulated using a number of synergistic ingredients known to have benefits in subjects with mild to moderate scalp psoriasis or SD using trichoscopy as well as subjective and objective assess- 
ments of changes in symptoms. The shampoo ingredients include ketoconazole $[28,29]$ which reduces Malassezia spp., numerous ingredients that promote hair growth [30,31], and a broad-spectrum CBD, which was added to the formulation for its anti-inflammatory effects on the pilosebaceous unit and potential to reduce oxidative stress. Cannabidiol is immunosuppressive for various immune cell types, inducing apoptosis and promotion of regulatory cells, which control other immune cell targets [32]. It reduces the expression of nitrotyrosine (iNOS) in different tissues suggesting they may be responsible for the reduction of cytokine production, counteracting the rise in iNOS levels and the downstream cascade of events triggered by the inflammatory process, and reducing oxidative stress [33]. They also inhibit lymphocyte proliferation and the production of interleukin-2 (IL-2), interferon-c, IL-1a, IL-1b, and tumor necrosis factor-alpha.

The interaction between the anti-inflammatory pathway and the endocannabinoid system suggests a role for cannabinoids in treating psoriasis [34]. Cannabidiol can exert a suppressive effect on human keratinocyte proliferation $[35,36]$. The antiproliferative and anti-inflammatory effects of CBD could be associated with specific sebostatic actions. It normalizes the pathologically elevated lipogenesis, suppresses cell proliferation, and prevents the actions of toll-like receptor activation that elevates proinflammatory cytokine levels [12]. Cannabidiol mayinhibit the enzyme 5- $\alpha$-reductase, subsequently inhibiting excessive skin sebum secretion and improving skin elasticity and hydration. Cannabidiol has high lipophilicity that helps it to reach and accumulate in the sebaceous glands through transfollicular delivery, having a therapeutic effect hours after applying the product [12].

In our study, the study shampoo containing a broadspectrum CBD was shown to be highly effective in reducing signs of scalp inflammation (arborizing vessels, twisted capillaries, and scales), as well as reducing symptoms of erythema and scaling, itching, and burning, following 2 weeks' use. Baseline severity was similar in males and females, and there was no significant gender difference in terms of reduction in itching and burning, tolerability, or subject satisfaction in males or females.

In conclusion, replacing current shampooing practices with a broad-spectrum cannabidiol-containing shampoo significantly reduces both severity and symptoms of scalp inflammation within 2 weeks, with excellent tolerability and treatment satisfaction in subjects with mild to moderate scalp psoriasis or SD.

\section{Statement of Ethics}

The EU guidelines on IRB approval (reinforced by Italian law regarding tests on human beings) exclude from IRB approval cosmetic testing as mentioned in section 1.2.9 of the Official Journal of EU (March 30, 2010). Anyway, we committed ourselves to the safeguard of the rights and well-being of all the trial subjects by following the principles of the Helsinki Declaration and letting them know all the relevant aspects of their participation through a written informed consent.

\section{Conflict of Interest Statement}

Dr. Vincenzi has no conflicts; Dr. Tosti is a consultant for DS Healthcare Group, Monat Global, Almirall, Thirty Madison, Lilly, Leo Pharmaceuticals, Bristol Myers Squibb, and P\&G.

\section{Funding Sources}

The study was funded by a grant from DS Laboratories.

\section{Author Contributions}

Dr. Vincenzi: collection and organization of data, and drafting of manuscript. Dr. Tosti: study protocol, revision of data, and final manuscript.

\section{References}

1 Bickers DR, Athar M. Oxidative stress in the pathogenesis of skin disease. J Invest Dermatol. 2006;126(12):2565-75.

2 Trueb RM, Henry JP, Davis MG, Schwartz JR Scalp condition impacts hair growth and retention via oxidative stress. Int J Trichology. 2018;10(6):262-70.

3 Bakry OA, Elshazly RM, Shoeib MA, Gooda A. Oxidative stress in alopecia areata: a casecontrol study. Am J Clin Dermatol. 2014; 15(1):57-64.
4 Yenin JZ, Serarslan G, Yönden Z, Ulutaş KT Investigation of oxidative stress in patients with alopecia areata and its relationship with disease severity, duration, recurrence and pattern. Clin Exp Dermatol. 2015;40(6):61721.

5 Crowley J. Scalp psoriasis: an overview of the disease and available therapies. J Drugs Dermatol. 2010;9(8):912-8.

6 Borda LJ, Wikramanayake TC. Seborrheic dermatitis and dandruff: a comprehensive review. J Clin Investig Dermatol. 2015;3(2):1-22. 
7 Lebwohl MG, Bachelez H, Barker J, Girolomoni G, Kavanaugh A, Langley RG, et al. Patient perspectives in the management of psoriasis: results from the population-based Multinational Assessment of Psoriasis and Psoriatic Arthritis Survey. J Am Acad Dermatol. 2014;70(5):871-30.

8 Hägg D, Sundström A, Eriksson M, SchmittEgenolf M. Severity of psoriasis differs between men and women: a study of the clinical outcome measure Psoriasis Area and Severity Index (PASI) in 5438 Swedish register patients. Am J Clin Dermatol. 2017;18(4):58390.

9 Sanders MGH, Pardo LM, Franco OH, Ginger RS, Nijsten T. Prevalence and determinants of seborrheic dermatitis in a middleaged and elderly population: the Rotterdam study. Br J Dermatol. 2018;178(1):148-53.

10 Golinska J, Sar-Pomian M, Rudnicka L. Dermoscopic features of psoriasis of the skin, scalp and nails: a systematic review. J Eur Acad Dermatol Venereol. 2019;33(4):648-60.

11 Miteva M, Tosti A. Hair and scalp dermatoscopy. J Am Acad Dermatol. 2012;67(5): $1040-8$.

12 Oláh A, Tóth BI, Borbíró I, Sugawara K, Szöllõsi $A G$, Czifra $G$, et al. Cannabidiol exerts sebostatic and antiinflammatory effects on human sebocytes. J Clin Invest. 2014;124(9): 3713-24.

13 Martinez-Velasco MA, Vazquez-Herrera NE, Tosti A. How to evaluate treatment response in hair diseases in hair and scalp treatments: a practical guide, chapter 20. In: Tosti A, AszSigall D, Pirmez R, editors. Springer Verlag; 2019. p. 291-322.

14 Kim TW, Shim WH, Kim JM, Mun JH, Song M, Kim HS, et al. Clinical characteristics of pruritus in patients with scalp psoriasis and their relation with intraepidermal nerve fiber density. Ann Dermatol. 2014;26(6):727-32.

15 Jung GW, Ko HJ, Kim HC, Lee MB, Lee WJ au SJ, et al. Dermoscopy can be useful in differentiating scalp psoriasis from seborrhoeic dermatitis. Br J Dermatol. 2011;164(3):652-6.

16 DeAngelis YM, Gemmer CM, Kaczvinsky JR, Kenneally DC, Schwartz JR, Dawson TL Jr. Three etiologic facets of dandruff and seborrheic dermatitis: Malassezia fungi, sebaceous lipids, and individual sensitivity. J Investig Dermatol Symp Proc. 2005;10(3):295-7.
17 Ozturk P, Arican O, Belge Kurutas E, Karakas T, Kabakci B. Oxidative stress in patients with scalp seborrheic dermatitis. Acta Dermatovenerol Croat. 2013;21(2):80-5.

18 Schwartz JR, Henry JP, Kerr KM, Mizoguchi $\mathrm{H}$, Li L. The role of oxidative damage in poor scalp health: ramifications to causality and associated hair growth. Int J Cosmet Sci. 2015; 37(Suppl 2):9-15.

19 Gomez-Moyano E, Crespo-Erchiga V, Martínez-Pilar L, Godoy Diaz D, Martínez-García S, Lova Navarro M, et al. Do Malassezia species play a role in exacerbation of scalp psoriasis? J Mycol Med. 2014;24(2):87-92.

20 Kadam DP, Suryakar AN, Ankush RD, Kadam CY, Deshpande KH. Role of oxidative stress in various stages of psoriasis. Indian $\mathrm{J}$ Clin Biochem. 2010;25(4):388-92.

21 Yazici C, Köse K, Utaş S, Tanrikulu E, Taşlidere N. A novel approach in psoriasis: first usage of known protein oxidation markers to prove oxidative stress. Arch Dermatol Res. 2016;308(3):207-12.

22 Dimon-Gadal S, Gerbaud P, Thérond P, Guibourdenche J, Anderson WB, Evain-Brion D, et al. Increased oxidative damage to fibroblasts in skin with and without lesions in psoriasis. J Invest Dermatol. 2000;114(5):984-9.

23 Bayer M, Mosandl A, Thaçi D. Improved enantioselective analysis of polyunsaturated hydroxy fatty acids in psoriatic skin scales using high-performance liquid chromatography. J Chromatogr B Analyt Technol Biomed Life Sci. 2005;819(2):323-8.

24 Squire RA, Goode K. A randomised, singleblind, single-centre clinical trial to evaluate comparative clinical efficacy of shampoos containing ciclopirox olamine (1.5\%) and salicylic acid (3\%), or ketoconazole (2\%, nizoral) for the treatment of dandruff/seborrhoeic dermatitis. J Dermatolog Treat. 2002;13(2): 51-60.

25 Piérard-Franchimont C, Goffin V, Decroix J, Piérard GE. A multicenter randomized trial of ketoconazole $2 \%$ and zinc pyrithione $1 \%$ shampoos in severe dandruff and seborrheic dermatitis. Skin Pharmacol Appl Skin Physiol. 2002;15(6):434-41.

26 Okokon EO, Verbeek JH, Ruotsalainen $\mathrm{JH}$ Ojo OA, Bakhoya VN. Topical antifungals for seborrhoeic dermatitis. Cochrane Database Syst Rev. 2015;5(5):CD008138.
27 van de Kerkhof PC, Franssen ME. Psoriasis of the scalp. Diagnosis and management. Am J Clin Dermatol. 2001;2(3):159-65.

28 Piérard-Franchimont C, Goffin V, Henry F, Uhoda I, Braham C, Piérard GE. Nudging hair shedding by antidandruff shampoos. A comparison of $1 \%$ ketoconazole, $1 \%$ piroctone olamine and $1 \%$ zinc pyrithione formulations. Int J Cosmet Sci. 2002;24(5):249-56.

29 Choi FD, Juhasz MLW, Atanaskova Mesinkovsks N. Topical ketoconazole: a systematic review of current dermatological applications and future developments. J Dermatog Treat. 2019;30(8):760-71.

30 Fischer TW, Herczeg-Lisztes E, Funk W, Zillikens D, Bíró T, Paus R. Differential effects of caffeine on hair shaft elongation, matrix and outer root sheath keratinocyte proliferation, and transforming growth factor- $\beta 2 /$ insulinlike growth factor-1-mediated regulation of the hair cycle in male and female human hair follicles in vitro. Br J Dermatol. 2014;171(5): 1031-43.

31 Trueb RH. Serum biotin kevels in women complaining of hair loss. Int J Trichology. 2016;8(2):73-7.

32 Nicols JM, Kaplan BLF. Immune responses regulated by cannabidiol. Cannabis Cannabinoid Res. 2002;5(1):12-31.

33 Szabó IL, Lisztes E, Béke G, Tóth KF, Paus R, Oláh A, et al. The phytocannabbinoid (-)-cannabidiol operates as a complex, differential modulator of human hair growth: anti-inflammatory submicromolar versus hair growth inhibitory micromolar effects. J Invest Dermatol. 2020;140(2):484-8.

34 Derakhshan N, Kazemi M. Cannabis for refractory psoriasis-high hopes for a novel treatment and a literature review. Curr Clin Pharmacol. 2016;11(2):146-7.

35 Stander S, Reinhardt HW, Luger TA. Topical cannabinoid agonists. An effective new possibility for treating chronic pruritus. Hautarzt. 2006;57(9):801-7.

36 Jordt SE, Bautista DM, Chuang HH, McKemy DD, Zygmunt PM, Högestätt ED, et al. Mustard oils and cannabinoids excite sensory nerve fibres through the TRP channel ANKTM1. Nature. 2004;427(6971):260-5.
Broad-Spectrum Cannabidiol Shampoo for Inflammatory Scalp Disorders
Skin Appendage Disord 2020;6:355-361 DOI: $10.1159 / 000510896$ 